\title{
1 Contact-dependent traits in Pseudomonas syringae B728a
}

2 Monica N. Hernandez ${ }^{1 *}, \# a$ and Steven E. Lindow ${ }^{1}$

3 'Department of Plant and Microbial Biology, University of California, Berkeley, California,

4 United States of America

$5 \quad{ }^{\# a}$ Current Address: Department of Botany and Plant Pathology, Southern Oregon Research and 6 Extension Center affiliated with Oregon State University, Central Point, Oregon, United States

7 of America

$9 *$ Monica N. Hernandez

10 E-mail: monica.hernandez@oregonstate.edu

11 Short Title: Contact-dependent traits

12

15 Abstract

16 Production of the biosurfactant syringafactin by the plant pathogen Pseudomonas syringae

17 B728a is a surface contact-dependent trait. Expression of $s y f A$, as measured using a $g f p$ reporter

18 gene fusion was low in planktonic cells in liquid cultures but over 4-fold higher in cells

19 immobilized on surfaces as varied as glass, plastic, paper, parafilm, agar, membrane filters, and

20 leaves. Induction of $s y f A$ as measured by GFP fluorescence was rapid, occurring within two

21 hours after immobilization of cells on surfaces. Comparison of the global transcriptome by RNA

22 sequencing of planktonic cells in a nutrient medium with that of cells immobilized for 2 hours on

23 filters placed on this solidified medium revealed that, in addition to $s y f A, 3156$ other genes were 
24 differentially expressed. Genes repressed in immobilized cells included those involved in

25 quaternary ammonium compound (QAC) metabolism and transport, compatible solute

26 production, carbohydrate metabolism and transport, organic acid metabolism and transport,

27 phytotoxin synthesis and transport, amino acid metabolism and transport, and secondary

28 metabolism. Genes induced in immobilized cells included $s y f A$ plus those involved in translation,

29 siderophore synthesis and transport, nucleotide metabolism and transport, flagellar synthesis and

30 motility, lipopolysaccharide (LPS) synthesis and transport, energy generation, transcription,

31 chemosensing and chemotaxis, replication and DNA repair, iron-sulfur proteins,

32 peptidoglycan/cell wall polymers, terpenoid backbone synthesis, iron metabolism and transport,

33 and cell division. That many genes are rapidly differentially expressed upon transfer of cells

34 from a planktonic to an immobilized state suggests that cells experience the two environments

35 differently. It seems possible that surface contact initiates anticipatory changes in $P$. syringae

36 gene expression, which enables rapid and appropriate physiological responses to the new

37 environmental conditions. Such responses could help cells survive transitions from aquatic

38 habitats fostering planktonic traits to attachment on surfaces, conditions that alternatively occur

39 on leaves.

\section{Introduction}

42 Pseudomonas syringae has adapted to live in a variety of different environments. While most

43 studies of this taxon have focused on its life as a plant colonist in which it grows on the surfaces

44 of leaves and subsequently in the apoplast where it can cause disease, many strains apparently

45 inhabit other habitats. For instance, Morris et al. [1] described P. syringae in snow, rain, and in

46 lakes and rivers, suggesting that this bacterium is disseminated through the water cycle. All 
47 bacteria are intrinsically aquatic, although many have adapted to survive in periodically dry

48 environments, often forming biofilms on surfaces in contact with water. The very different

49 chemical and physical properties of aquatic environments versus surfaces, which in the case of $P$.

50 syringae are frequently dry, presumably select for coordinated gene expression that optimizes

51 fitness across these different conditions. Leaf surfaces are relatively harsh habitats that exhibit

52 strong spatially and temporally varying conditions. Water and nutrients are unevenly dispersed

53 across leaf surfaces [2,3] and leaves experience rapid temporal fluctuations in temperature,

54 humidity, and liquid water availability [3]. Leaf surfaces also experience high ultraviolet

55 radiation flux [3]. In a bacterial population inhabiting such a setting, some cells might exhibit a

56 planktonic existence within water drops containing nutrients, while other cells must contend with

57 relatively dry surfaces.

58 Previous studies have revealed the role of syringafactin, a hygroscopic biosurfactant whose

59 production by $P$. syringae is encoded by $s y f A$, as an adaptation to the frequent absence of liquid

60 water on leaf surfaces [4,5]. By binding water vapor trapped within the humid boundary layer

61 and/or by binding liquid water that is transiently present on leaves, syringafactin can alleviate

62 water stress by expanding the conditions under which liquid water is available for P. syringae [6].

63 Furthermore, syringafactin does not seem to disperse in water, perhaps due to its strong

64 hydrophobic characteristics, but instead remains immobilized onto leaf surfaces near the cells

65 that produced it [5]. Syringafactin is thus unlikely to benefit cells immersed in water, such as

66 those occurring in the planktonic state in aquatic environments involved in the water cycle [1].

67 Instead, syringafactin production might maximally benefit cells in non-aquatic settings where it

68 would improve $P$. syringae survival on surfaces subject to frequent drying. Interestingly, Burch

69 et al. [7] found that cells grown on agar surfaces produced much more syringafactin and 
expressed $s y f A$ at a much higher level than those in broth cultures. This observation suggests two competing hypotheses. First, $s y f A$ differential gene expression may be instigated by the

72 differences in chemical or physical conditions found on an agar surface compared to that in a

73 similar liquid culture. Alternatively, immobilization of cells on a surface may be used as a cue to

74 immediately indicate the current or anticipated presence of environmental conditions common to

75 leaf surfaces where the production of syringafactin would be maximally beneficial to the cell.

76 Since Burch et al. [7] examined syfA expression only after growth of bacteria in different

77 conditions for extended time periods ( $>24$ hours), they could not unambiguously distinguish

78 between these two models of gene regulation.

79 To distinguish between these two hypotheses, we determined how quickly $s y f A$ expression

80 increased after planktonic cells of $P$. syringae contacted a surface and whether the nature of the

81 surface it interacted with determined the extent of any response. It seemed likely that cells of $P$.

82 syringae might benefit from anticipatory patterns of gene expression, where certain cues might

83 be used to indicate large and rapid changes in environmental conditions. Thus, rapid or prior

84 expression of certain traits would be highly beneficial in adapting to large changes in habitat

85 characteristics. In this case, we predicted that changes in gene expression associated with

86 transition from a planktonic to a surface-associated habitat such as a leaf would be linked to

87 perception of a surface that might be imminently dry. While there has been interest in such

88 surface sensing in bacteria $[8,9,10,11,12]$ little is known of the mechanism by which cells

89 sense surfaces and there are few studies that have examined rapid changes in gene expression

90 that could be associated with perception of a surface [13]. We therefore explored the rapid

91 changes in the global transcriptome of B728a soon after planktonic cells became sessile on a

92 surface. The large number of genes that we find to undergo changes in gene expression upon the 
93 transition from planktonic to sessile life suggest that surface sensing could be a major cue

94 controlling adjustment of $P$. syringae to the varying habitats it colonizes on leaf surfaces and

95 elsewhere.

\section{Materials and methods}

\section{Bacterial strains and growth conditions}

Pseudomonas syringae B728a strains were grown on King's medium B (KB) plates containing

$991.5 \%$ technical agar or in KB broth lacking agar [14]. Antibiotics were used at the following

100 concentrations $(\mu \mathrm{g} / \mathrm{ml})$ : spectinomycin (100), kanamycin (50), and tetracycline (15) as

101 appropriate.

\section{Quantification of GFP fluorescence in individual bacterial cells}

103 P. syringae B728a cells harboring a plasmid in which the $s y f A$ promoter was fused to a

104 promoterless $g f p$ reporter gene [7], were grown in KB broth until they reached a density of $10^{8}$

105 cells $/ \mathrm{ml}$ as determined by Optical Density $(\lambda=600 \mathrm{~nm}) .10 \mu 1$ of cells were then placed onto

106 various surfaces. To subsequently remove cells from these surfaces, moistened cotton swabs

107 were used to scrub off the cells. The swabs were then placed into microcentrifuge tubes

108 containing $100 \mu \mathrm{l}$ of water and vortexed to re-suspend the cells. $5 \mu \mathrm{l}$ of cell suspension from

109 each treatment was applied to glass slides for fluorescence microscopy to quantify the GFP

110 fluorescence of individual cells. Cells were visualized at 100X magnification with a M2

111 AxioImager. A GFP filter set was used to view cells, and images were captured in black and

112 white format using a 12-bit Retiga camera. The software iVision was used to identify all 
113 bacterial cells in an image and to quantify the average pixel intensity of each object identified as

114 in other studies [6]. Aggregates of bacterial cells and extraneous particles were identified by

115 visual examination and marked for exclusion before image processing.

\section{RNA isolation}

117 Bacterial cells were grown with shaking in $\mathrm{KB}$ broth at $28^{\circ} \mathrm{C}$ until cultures reached a cell density 118 of $5 \times 10^{8}$ cells $/ \mathrm{ml}$. Three replicate cultures were used. $300 \mu 1$ of each culture was then applied to 119 a $0.4 \mu \mathrm{m}$ Isopore ${ }^{\circledR}$ membrane filter and excess liquid was removed by exposing the filter to a

120 vacuum source for 5 seconds. Filters were then immediately placed onto KB plates to incubate at $12128^{\circ} \mathrm{C}$ for two hours while broth cultures were returned to a shaker and incubated at $28^{\circ} \mathrm{C}$ for two 122 more hours. Cells in broth cultures were harvested by pipetting $1 \mathrm{ml}$ of suspension into a $15 \mathrm{~mL}$ 123 conical tube containing $125 \mu \mathrm{l}$ ice-cold EtOH/Phenol stop solution (5\% water-saturated phenol

$124(\mathrm{pH}<7.0)$ in ethanol). Filters were immersed in the EtOH/Phenol stop solution and cells were 125 harvested by sonication for 30 seconds followed by vortexing for 20 seconds to ensure complete 126 cell detachment from the filters. Cells were then collected by centrifugation at 12,000 rpm

$127(13,800 \mathrm{x} g)$ for five minutes at $4^{\circ} \mathrm{C}$. Supernatant was decanted and the cells were frozen in 128 liquid nitrogen and stored at $-80^{\circ} \mathrm{C}$ until RNA isolation. RNA isolation was performed using a 129 Direct-zol ${ }^{\mathrm{TM}}$ RNA Kit (Zymo Research). To isolate RNA, $600 \mu$ l of TRI Reagent ${ }^{\circledR}$ (Sigma130 Aldrich) was then added to the pelleted cells. An equal volume of ethanol (100\%) was added to 131 the cells in TRI Reagent ${ }^{\circledR}$ and mixed thoroughly. The mixture was transferred into a Zymo132 Spin ${ }^{\mathrm{TM}}$ IICG Column (Zymo Research) in a collection tube and centrifuged. The flow-through 133 was discarded and the column was transferred into a new collection tube. For DNase I treatment, $134400 \mu 1$ RNA Wash Buffer (Zymo Research) was added to the column which was then 135 centrifuged. $5 \mu 1$ DNase I (6 U/ $\mu 1)$ (Zymo Research) and $75 \mu 1$ DNA Digestion Buffer (Zymo 
136 Research) were then added to the tube and mixed. The mix was added directly to the column

137 matrix and incubated at room temperature $\left(20-30^{\circ} \mathrm{C}\right)$ for 15 minutes. $400 \mu 1$ Direct-zol ${ }^{\mathrm{TM}} \mathrm{RNA}$

138 PreWash (Zymo Research) was then added to the column and then centrifuged. The flow-

139 through was discarded and the step was repeated once more. $700 \mu 1$ RNA Wash Buffer (Zymo

140 Research) was then added to the column and subsequently centrifuged for two minutes. The

141 column was then transferred into a RNase-free tube. To elute RNA, $50 \mu 1$ of DNase/RNase-Free

142 Water (Zymo Research) was added directly to the column matrix which was then centrifuged.

143 RNA was stored frozen at $-80^{\circ} \mathrm{C}$.

\section{5 mRNA sequencing}

$1461 \mu \mathrm{l}$ of each sample was diluted into $4 \mu \mathrm{l}$ of RNase free water and submitted to the Vincent J.

147 Coates Genomics Sequencing Laboratory at UC Berkeley where Ribo-Zero was used for rRNA

148 removal. RNA abundance and purity were determined using a 2100 Bioanalyzer (Agilent

149 Technologies) and quantified using Qubit (Invitrogen). After reverse transcription, shearing of

150 cDNA, size fractionation, and Illumina library production, the Vincent J. Coates Genomics

151 Sequencing Laboratory samples were sequenced using an Illumina HiSeq4000 platform with 50

152 base pair, single-end reads. Three biological replicates were sequenced per treatment. Reads

153 were uploaded to Galaxy [15] and cleaned using Trimmomatic [16]. Reads were aligned to the

154 Pseudomonas syringae B728a genome [17] using Salmon Transcript Quantification [18] in

155 Galaxy. The program edgeR in $\mathrm{R}$ was then used to assess the statistical significance of

156 differential gene expression [19]. Gene expression levels were normalized using a weighted

157 trimmed mean of $\mathrm{M}$ values (TMM; where $\mathrm{M}$ is the log expression ratio per gene between

158 treatments) [20]. Empirical Bayes estimation and tests based on the negative binomial 
159 distribution were then used to determine significance [20]. A gene was considered significantly

160 differentially regulated if the $\mathrm{p}$-value for the difference in relative expression between the filter

161 treatment and the liquid treatment was less than 0.001.

162

163 Statistical analysis

164 The hypergeometric distribution was performed in R [21] to test for significance of functional 165 category enrichment [22]. All p-values were adjusted using the Bonferroni correction [23] and 166 the Benjamini-Hochberg correction [24] in R.

\section{Results}

\section{$169 \operatorname{Sy} \boldsymbol{f} A$ induction occurs rapidly upon surface contact}

170 If the $s y f A$ gene is regulated directly as a response to contact with a surface, we would expect

171 gene induction to occur shortly after surface contact. To test how rapidly $s y f A$ was induced after

172 planktonic cells were transferred to various surfaces, we monitored the expression of syfA in $P$.

173 syringae B728a harboring a plasmid containing the $s y f A$ promoter fused to a promoterless $g f p$

174 reporter gene by assessing the GFP fluorescence of individual cells by epifluorescence

175 microscopy. Planktonic cells from broth cultures were applied to three surfaces: (1) agar-

176 solidified King's medium B (KB) media, (2) a $0.4 \mu \mathrm{m}$ polycarbonate Isopore ${ }^{\circledR}$ filter that was

177 placed on KB agar, and (3) a $0.4 \mu \mathrm{m}$ Isopore ${ }^{\circledR}$ filter floated on the surface of a small quantity of

$178 \mathrm{~KB}$ broth. Since nutrient solution diffused through the filters, wetting the filters in these settings,

179 we presume that the cells experienced similar levels of most nutrients while immobilized on the 
180 filters as those remaining in broth cultures but experienced different physical conditions.

181 Planktonic cells that remained in broth cultures served as a control. We compared gene

182 expression of cells on filters placed on KB agar and KB broth to test whether chemical

183 components of the agar, rather than simply the physical change it elicited, contributed to

184 induction of $s y f A$ expression as seen by Burch et al. [7]. By two hours after transfer to each of

185 the solid surfaces, cells exhibited significantly greater GFP fluorescence than those remaining in

186 the broth cultures (Fig 1). Similar levels of syfA induction occurred on all surfaces (Fig 1). Thus,

187 syfA induction clearly occurs rapidly upon encountering a surface and elevated expression on

188 agar appears to be due to the exposure of cells to a surface rather than any chemical components

189 of the agar.

190 Fig 1. Induction of syf A expression in cells of Pseudomonas syringae B728a as a function of

191 time.

192 Gene expression is estimated as the mean GFP fluorescence of individual cells harboring a

193 plasmid in which the $s y f A$ promoter was fused to a $g f p$ reporter gene when harvested from these

194 surfaces at the various times shown on the abscissa. 7,038 cells were evaluated in total for

195 control cells that remained in a broth culture (blue); 3,068 cells were evaluated after application

196 to agar (green); 10,184 cells were evaluated after application to filters on an agar surface

197 (yellow); and 3,791 cells were evaluated after application to a filter placed on plastic (orange).

198 The vertical bars represent the standard error of the mean GFP fluorescence for a given cell.

$199 \boldsymbol{S y} \boldsymbol{f} \boldsymbol{A}$ is induced in cells on a variety of solid surfaces

200 Given that syfA induction quickly followed transfer of planktonic cells to various surfaces with

201 abundant nutrient resources, we explored whether any particular physical parameters such as 
202 hydrophobicity or roughness were associated with the induction process. Since $P$. syringae

203 B728a was isolated from leaf surfaces and is highly fit as an epiphyte $[25,26]$, we tested the

204 hypothesis that leaf surfaces would confer the most rapid or largest contact-dependent induction

205 of this gene. By exposing cells to various types of solid surfaces we further explored whether

$206 s y f A$ expression was regulated by contact with a surface per se as opposed to a chemical cue.

207 Planktonic cells from KB broth cultures were applied to polycarbonate, paper, parafilm, excised

208 leaves, water agar, and Isopore ${ }^{\circledR}$, Durapore ${ }^{\circledR}$, and Teflon ${ }^{\circledR}$ filters. Cells all exhibited at least 2-

209 fold greater GFP fluorescence than those of the planktonic cells when assessed six hours after

210 application to these surfaces (Fig 2). Little difference in GFP fluorescence was observed among

211 cells placed on the various solid surfaces, suggesting that neither surface type-dependent

212 chemical cues nor the physical properties of the surface on which cells were immobilized

213 strongly influenced the induction of $s y f A$.

\section{Fig 2. The expression of $\boldsymbol{s y} \boldsymbol{A} \boldsymbol{A}$ increases rapidly after immobilization of cells of Pseudomonas}

\section{5 syringae $\mathrm{B} 728 \mathrm{a}$.}

216 Gene expression is estimated as the mean GFP fluorescence of individual cells harboring a

217 plasmid in which the $s y f A$ promoter was fused to a $g f p$ reporter gene when harvested 6 hours

218 after application to glass (green), polycarbonate plastic (yellow), paper (orange), parafilm (red),

219 bean leaves (purple), agar (grey), Isopore filters (pink), Durapore filters (aqua), Teflon filters

220 (brown), or recovered from broth cultures at the time of application to surfaces or after 6 hours

221 (blue). The vertical bars represent the standard error of the mean GFP fluorescence for a given

222 cell. 


\section{RNA sequencing of $\boldsymbol{P}$. syringae B728a reveals many genes}

\section{5 differentially regulated in cells transferred to a filter surface versus}

\section{6 in liquid culture}

227 Given that cells of $P$. syringae exhibited large increases in $s y f A$ expression within two hours of

228 transfer to a variety of solid surfaces, we performed RNA sequencing to determine the extent to

229 which other genes exhibit contact-dependent gene expression. The transcriptome of cells grown

230 in KB broth was compared to those placed on filters atop KB agar for 2 hours. RNA sequencing

231 returned between 20 and 40 million reads per sample (Fig S1). As expected, a wide range of

232 reads were recovered for a given gene (Fig S2). A heatmap revealed that while patterns of gene

233 regulation were similar among replicates in which cells were either planktonic or immobilized on

234 a filter, gene expression differed strongly between cells in these two conditions (Fig 3). Much of

235 the variation in relative gene expression levels of the average gene was thus associated with the

236 environment of the cells before RNA was harvested rather than other factors.

238 Fig 3. Heat map illustrating different patterns of gene expression in cells of Pseudomonas

239 syringae B728a.

240 Heat map illustrating different patterns of gene expression in cells of Pseudomonas syringae

241 B728a 2 hours after application to a filter surface compared to that in broth culture. Differential

242 up-regulation of genes is shown in yellow while down-regulation is depicted in red.

244 Expression of 1,390 genes was up-regulated in cells on the filter surface compared to those in

245 broth media, while expression of 1,766 genes was down-regulated on filter surfaces compared to 
246 that in broth (Fig 4). Of the 1,390 genes that were induced on filter surfaces, 881 were induced

247 more than 2-fold while 509 exhibited lesser induction. Of the 1,766 genes that were repressed on

248 filter surfaces, 1,138 were repressed more than 2-fold while 628 were repressed less than 2-fold.

249 In total, 3,156 genes $(60.46 \%$ of the $P$. syringae B728a genome) were found to be differentially

250 expressed in cells on the filter surface compared to those in broth culture (Fig 4). There was no

251 apparent relationship between the absolute level of expression of a gene and the likelihood that it

252 would exhibit differential expression in these two settings (Fig S3).

Fig 4. Volcano plot illustrating the differential expression of genes in Pseudomonas syringae

255 B728a. Volcano plot illustrating the differential expression of genes in Pseudomonas syringae

256 B728a 2 hours after application to a filter surface. Genes that are significantly differentially

257 expressed compared to that in broth culture are shown in red, and those that do not differ are

258 shown in black.

\section{Functional category analysis}

261 We grouped the differentially regulated genes into functional categories to better establish those

262 processes that were most differentially expressed in planktonic and immobilized cells (Tables 1

263 and 2). Twice as many functional gene categories were enriched in genes that were significantly

264 up-regulated in immobilized cells than those that were down-regulated. The following gene

265 functional categories were examined in further detail: 
Gene Category

Bonferroni adjusted Benjamini-Hochberg adjusted p-

p-value

1.11E-18

4.75E-09

2.38E-09

transport

Nucleotide metabolism and

transport

Flagellar synthesis and

motility

value

1.11E-18

Siderophore synthesis and

1.12E-06

3.73E-07
Lipopolysaccharide synthesis
9.16E-03
1.83E-03

and transport

Energy generation

0.02

2.63E-03

Transcription

0.04

0.04

Chemosensing and

chemotaxis

0.06

7.15E-03

Replication and DNA repair

0.12

0.01

Iron-sulfur proteins

0.19

0.02 


\section{Peptidoglycan/cell wall}

polymers
0.19
0.02

Terpenoid backbone

synthesis

$\begin{array}{ll}0.24 & 0.02\end{array}$

Iron metabolism and

$\begin{array}{ll}0.25 & 0.02\end{array}$

transport

Cell Division

0.59

0.04

270 The significance of functional category enrichment was assessed using the hypergeometric distribution

271 (Castillo-Davis and Hartl, 2003).

272

273

274

275

276

277

278

279

280 
Table 2. Functional gene categories of Pseudomonas syringae B728a preferentially down-

\section{Gene Category}

\section{Bonferroni adjusted}

p-value
Benjamini-Hochberg

adjusted $p$-value
Quaternary ammonium

compound metabolism and

transport
2.19E-08

2.19E-08

Compatible solute synthesis

2.33E-06

1.17E-06

Carbohydrate metabolism and

transport

1.72E-05

5.74E-06

Organic acid metabolism and

transport

8.84E-05

2.21E-05

Phytotoxin synthesis and

transport

\subsection{E-04}

2.32E-05

Amino acid metabolism and

transport

0.01

1.77E-03

Secondary metabolism

0.01

1.01E-03

284 The significance of functional category enrichment was assessed using the hypergeometric distribution

285 (Castillo-Davis and Hartl, 2003). 
286 Translation. Many genes encoding the 30S and 50S ribosomal protein subunits were induced

287 on the filter surface, as were genes encoding the elongation factor proteins $\mathrm{Ts}, \mathrm{P}, \mathrm{Tu}$, and $\mathrm{G}$.

288 Many genes encoding t-RNA synthetases were also induced. It thus appeared that translation as a

289 whole may have accelerated upon transition of cells from a planktonic to an immobilized state.

290 Siderophore synthesis and iron metabolism. Many genes involved in siderophore

291 synthesis and transport were induced on the filter surface. Many genes such as $p v d S, p v d G, p v d L$,

$292 p v d I, p v d J, p v d K, p v d D, p v d E, p b d O, p v d N, p v d T$, and $p v d R$ were involved in regulation of

293 pyoverdine production and its transport. Many genes involved in achromobactin regulation,

294 synthesis, and transport including $a c s G, a c s D, a c s E, y h c A, a c s C, a c s B, a c s A, \operatorname{car} A-2, \operatorname{cbr} B-2$,

295 and $c b r C$-2 were also induced. Many genes involved in iron metabolism and transport were also

296 induced on the filter surface. This included the genes $f e c E, f e c D, f e c C, f e c B, f e c A, f e c R$, and the

297 RNA polymerase ECF sigma factor fecI. These results suggest that iron became less available on

298 the filter surfaces than in broth, perhaps due to diffusional limitations associated with the lack of

299 mixing of cells as would occur in a liquid medium. Alternatively, iron might commonly be less

300 available on the natural surfaces on which $P$. syringae typically inhabits, such as leaf surfaces,

301 and the filter mimicked physical cues that the bacteria might use to anticipate transition into such

302 low iron environments.

303 Nucleotide metabolism and transport. Numerous genes involved in nucleotide

304 metabolism and transport were induced in immobilized cells. This included many genes involved

305 in purine and pyrimidine metabolism such as purA, purT, purC, purF, purB, purM, purN, purU-3,

306 purH, purD, purK, purE, and pyrB, pyrR, pyrH, pyrG, pyrF, pyrD, pyrC-2 respectively. As with

307 the apparent increase in translational activity seen upon transition of planktonic cells to those on 
surfaces, increased transcription might be expected to also be linked to such increases, requiring

310 involved in cell division were also induced on the filter surface. This included the cell division

311 proteins FtsK, FtsQ, and FtsL as well as the rod-shape determining proteins MreD and MreC.

$312 \mathrm{MrdB}$, a cell cycle protein, was also induced.

313 Flagellar synthesis and motility. Many genes encoding flagellar biosynthesis proteins,

314 flagellar basal body proteins, and flagellar hook-associated proteins were induced on the filter

315 surface. The gene encoding the anti-sigma-28 factor FlgM was also induced more than 2-fold.

316 Genes encoding the flagellar motor proteins MotA, MotB, MotC, and MotD were induced as

317 well. While it would be expected that planktonic cells of $P$. syringae would be motile, higher

318 levels of expression of motility genes on leaves compared to that in broth cultures has been

319 previously noted [26] and would likely require higher levels of flagellar production and repair [9].

320 Lipopolysaccharide synthesis and transport. All the genes with significant differential

321 expression that are involved in lipopolysaccharide (LPS) synthesis and transport were induced in

322 cells immobilized on filters except $\operatorname{arnB}$ and $\operatorname{arn} A$. Most of these induced genes are involved in

323 LPS transport and lipid A biosynthesis.

324 Energy generation. Many genes encoding proteins involved in oxidative phosphorylation

325 were expressed at a higher level on filter surfaces than in broth cultures. Such genes included

$326 c y o A, c y o B, c y o C$, and $c y o D$, all of which encode cytochrome c oxidase subunits, as well as $c c o N$,

$327 c c o O$, and $c c o P$ which encode cytochrome c oxidase cbb3-type subunits. Many genes encoding

328 F0F1 ATP synthase subunits were induced as well. 
329 Transcription. Similar to that seen for nucleotide synthesis, nearly all of the genes involved

330 in transcription that were differentially expressed were induced on filters compared to that in

331 broth cultures, with the exception of Psyr_4263. Genes up-regulated on surfaces included those

332 encoding the transcription termination factor Rho, the transcription elongation factors GreB and

333 GreA, the transcription anti-termination proteins NusB and NusG, and the DNA-directed RNA

334 polymerase subunits RpoA, RpoC, and RpoB.

335 Chemosensing and chemotaxis. Many genes involved in chemosensing and chemotaxis

336 were induced in cells attached to on the filter surface. Many of the induced genes encoded

337 histidine kinases. Interestingly, Psyr_1306, Psyr_1307 and Psyr_1308, which encode homologs

338 to $\mathrm{WspD}, \mathrm{WspE}$, and $\mathrm{WspF}$ respectively, were all down-regulated on the filter surface.

339 Psyr_1309, which encodes the homolog for WspR, was also down-regulated. However, this latter

340 gene was assigned to the cyclic diguanylate (cyclic di-GMP) cyclase proteins functional group.

341 Replication and DNA repair. Most of the genes involved in replication and DNA repair

342 were induced on the filter surface. This included Pysr_1408, Psyr_1409, and Psyr_1410 which

343 encode RuvC, RuvA, and RuvB respectively. These genes are also involved in homologous

344 recombination in addition to DNA repair.

345 Iron-sulfur proteins. Many genes encoding iron-sulfur proteins were induced on the filter

346 surface. This included $d s b E$ which is involved in cytochrome synthesis.

347 Peptiodoglycan/cell wall polymers. All of the genes involved in encoding

348 peptidoglycan/cell wall polymers that were differentially expressed on filters compared to broth 
349 cultures were induced on the filter surface. Most of these genes are involved in peptidoglycan

350 biosynthesis.

351 Terpenoid backbone synthesis. Many genes involved in terpenoid backbone synthesis

352 were induced in cells applied to filter surfaces. This included genes that are part of the

353 deoxyxylulose pathway of terpenoid biosynthesis.

354 Quaternary ammonium compound metabolism and transport. Surprisingly, all

355 genes with differential expression that are required for quaternary ammonium compound (QAC)

356 metabolism and transport were repressed on the filter surface. This included genes encoding

357 proteins involved in glycine betaine, choline, and carnitine metabolism and transport. The gene

358 betI, encoding the transcriptional regulator of choline degradation, was also down-regulated.

359 Similar to that seen for genes involved in QAC metabolism, all of the genes that had significant

360 differential expression that are involved in compatible solute synthesis were repressed on the

361 filter surface. Many of these genes contribute to either trehalose or N-acetylglutaminylglutamine

362 amide (NAGGN) synthesis. These compounds are solutes that are part of the cellular response to

363 water stress.

365 Carbohydrate metabolism and transport. Many of the genes involved in carbohydrate

366 metabolism and transport were repressed in immobilized cells on filters. This included genes

367 involved in trehalose, mannose, fructose, ribose, arabinose, maltose, manitol, and sorbitol

368 transport as well as genes involved in the pentose phosphate pathway. 
370 Organic acid metabolism and transport. Many of the genes involved in organic acid

371 metabolism and transport were also repressed on the filter surface. This included the genes $p h n F$,

$372 p h n G, p h n H, p h n I, p h n J, p h n K, p h n L, p h n M, p h n N$, and $p h n P$ which are all involved in

373 phosphonate metabolism and transport. The transcriptional regulator of vanillate metabolism,

374 vanR, was also down-regulated.

375 Phytotoxin synthesis and transport. All of the differentially expressed genes involved in

376 phytotoxin synthesis and transport were repressed in cells applied to filters. These genes included

377 salA, which is the regulator of syringomycin, as well as slyA, the regulator of syringolin A

378 production. Other genes involved in syringolin synthesis and transport $(\operatorname{sly} B, \operatorname{sly} C$, sly $D$, and

379 slyE), syringomycin synthesis and transport (syrE, syrC, syrB 1, syrP, and $s y r D)$, and

380 syringopeptin synthesis and transport ( $\operatorname{sy} A, \operatorname{syp} B$, and $s y p C$ ). Syringomycin and syringopeptin

381 secretion proteins PseA and PseB were also down-regulated in immobilized cells on filters.

382 Amino acid metabolism and transport. Many of the genes involved in amino acid

383 metabolism and transport were repressed on the filter surface. This included genes involved in

384 gamma-aminobutyric acid (GABA) metabolism (gabT-2, gabD-2, gabT-1, gabD-1, gabD-3, and

$385 g a b P)$.

387 Secondary metabolism. All of the differentially expressed genes involved in secondary

388 metabolism were repressed on the filter with the noteworthy exception of Psyr_2575, Psyr_2576,

389 and Psyr_2577 which encode SyfR, SyfA, and SyfB, responsible for the regulation of and

390 production of syringafactin, respectively. It was therefore intriguing to find that syringafactin 
391 production was the sole example of secondary compounds that were not down-regulated when

392 cells transitioned from a planktonic to a sessile state.

\section{Discussion}

394 The remarkably strong and rapid induction of syfA in P. syringae B728a in cells transferred from

395 broth culture to any of several different types of surfaces encouraged us to test the hypothesis

396 that a variety of other traits would exhibit similar surface-dependent changes in expression. The

397 rapidity with which $s y f A$ induction occurred on all of the various surfaces makes it unlikely that

398 cells modified their local microhabitat in any substantial way. We thus presume that assessment

399 of transcriptional changes within two hours after transfer to these surfaces reflected contact-

400 specific gene regulation rather than changes responsive to altered microenvironments that have

401 been seen in studies of biofilms. Such studies typically examined bacteria many hours after

402 attachment, usually after a thick biofilm had formed on surfaces [27, 28, 29]. We also presume

403 that regulatory shifts that occurred shortly after contact with a surface also were distinct from

404 those conditioned by other secondary events such as cell-cell contact or cell density-dependent

405 regulatory processes. Given the brief period of immobilization of cells on a filter surface, we

406 were surprised to find such a high proportion of the genes in P. syringae B728a to respond to this

407 transition. In addition to induction of the genes conferring syringafactin production and its

408 regulation ( $s y f A, s y f B$, and $s y f R$ ), it was striking that genes involved in many other cellular

409 processes such as flagellar synthesis and motility, LPS synthesis and transport, chemosensing

410 and chemotaxis, siderophore synthesis and transport, and DNA replication and repair underwent

411 changes in expression. While coherent arguments could be made for why some of these

412 processes should exhibit contact-dependent expression, the responses of many other processes 
413 remain enigmatic. The induction of many genes required for the synthesis of peptidoglycan/cell

414 wall polymers and LPS synthesis and transport seen in the immobilized cells (Table 1) suggests

415 that, like that of other bacteria for which surface contact-dependent gene expression has been

416 investigated [30, 31], P. syringae may also utilize a mechanosensitive pathway for sensing a

417 surface that involves disruption and repair of the cell envelope. Previous studies have suggested

418 that cells experience cell wall damage when they contact a surface [8,30]. Peptidoglycan and

419 cell wall synthesis might be induced to repair any damage that resulted from such an encounter.

420 Moreover, the bacterial outer membrane also contains abundant lipopolysaccharides that could

421 also be disrupted during physical binding of cells to a hard surface, making LPS synthesis

422 essential $[9,32,33]$. Interestingly, this initial cell wall stress may also be a cue for the

423 differential expression of other surface-regulated genes as suggested by others [8].

424 Historically, it has been common to study bacteria in broth cultures. While it is presumed that

425 such cultures are more homogeneous and facilitate studies of coordinated patterns of gene

426 expression etc., many aspects of the manner in which microorganisms interact with their

427 environment cannot be studied in such a setting $[8,11,12]$. For example, low nutrient conditions

428 in broth cultures are typically associated with both high cell densities and low oxygen levels. In

429 contrast, cells on the leaf surface would typically experience low nutrient conditions in a fully

430 aerobic environment. Although $P$. syringae B728a can be found in aquatic environments, it also

431 colonizes the surface of leaves, a decidedly different environment where it encounters both low

432 nutrient concentrations and high oxygen levels [1, 3, 34]. Furthermore, the study of bacterial

433 adaptations to life on surfaces has focused almost entirely on the process of biofilm formation in

434 aquatic settings $[27,28,29]$. The three-dimensional aggregates of bacteria that often form in

435 such aqueous environments, in which nutrients are provided by flowing liquids, are almost 
436 certainly very different from the monolayers of bacteria that typically develop on leaf surfaces

$437[35,36]$. Delivery of soluble nutrients in flowing liquids is probably an exceptional situation on

438 leaves [2, 3]. In addition, biofilm formation in a flowing liquid environment occurs over

439 relatively long periods of time. Cells are acquired by, or develop within, a biofilm over a long

440 period of time, during which the nature of the environment within the biofilm changes

441 dramatically, with large spaciotemporal variations [27]. For both practical and other reasons,

442 studies of aquatic biofilms typically have examined gene regulation only 24 hours or later after

443 biofilm initiation $[27,29]$. Few studies have addressed very early stages of biofilm formation

444 such as this study.

445 Genes involved in both transcription and translation were typically induced upon cell

446 immobilization (Table 1). Given that the transcription of a large number of genes was rapidly

447 increased upon transitioning from a planktonic to a sessile state (Figs 3 and 4), there would need

448 to be a corresponding increase in translation to produce the corresponding proteins. Likewise,

449 genes linked to nucleotide metabolism and transport, transcription, and replication and DNA

450 repair (Table 1) were also induced upon immobilization of cells. It would follow that higher

451 rates of nucleotide synthesis or procurement would be needed to support RNA synthesis

452 associated with the elevated transcription. The apparent elevated DNA replication associated

453 with cell immobilization would also require increased expression of genes enabling nucleotide

454 metabolism.

455 Studies have suggested that cells on leaf surfaces and attached to apoplastic surfaces experience

456 oxidative stresses resulting from plant defenses [37, 38, 39]. These compounds, including

457 hydroxyl radicals created when iron reacts with $\mathrm{H}_{2} \mathrm{O}_{2}$, can be damaging to iron-sulfur proteins

458 and DNA [39]. Genes involved in iron-sulfur protein synthesis and DNA repair would be 
expected to be induced on surfaces, as we observed here, if cells anticipated such a chemical assault on plant surfaces and linked their expression with surfaces per se.

461 Genes required for siderophore synthesis and transport as well as iron metabolism and transport, typically activated under conditions of low iron availability [26] were also induced in

463 immobilized cells (Table 1), suggesting that iron was less available to cells on the filter surface

464 than in broth media. Access to the limited iron in any environment would be expected to be 465 determined by its delivery to the vicinity of the cell by diffusion. Such a process would be 466 diminished in the two-dimensional environment on the surface of filters compared to the three467 dimensional habitat of cells immersed in a liquid medium. Cells affixed to a surface also would 468 be unable to move in response to local resource depletion to gain access to such molecules [13].

469 Given that iron is commonly present in low amounts on the natural surfaces that $P$. syringae 470 typically inhabits, such as leaves $[40,41]$ immobilization of cells on filters might have mimicked 471 this physical cue to anticipate transition into such low-iron environments.

472 The many genes involved in flagellar synthesis and motility and chemosensing and chemotaxis

473 that were surface induced (Table 1) would be important in the colonization of leaf surfaces that

474 harbor limited amounts of nutrient resources that are also heterogeneously dispersed [2, 42, 43].

475 Moreover, pathogenic bacteria such as $P$. syringae often eventually colonize the leaf apoplast - a

476 process requiring cells to move towards and enter a stomate or breaks in the cuticular surface of

477 leaves to access this intercellular habitat [34]. The up-regulation of motility and chemotaxis

478 genes was also observed in a study examining the transcriptome of $P$. syringae B728a on leaf

479 surfaces and apoplast when compared to that in a liquid medium in vitro [26]. Direct support for

480 the importance of cell motility of $P$. syringae on leaves was provided by studies of Haefele and

481 Lindow [43] who showed that non-motile mutants were much less fit than motile cells. 
482 Surprisingly, genes involved in QAC metabolism and compatible solute synthesis tended to be

483 repressed in cells on filter surfaces (Table 2). Such compounds often serve as compatible solutes

484 whose purpose is to maintain equilibrium of water availability between the inside of the cell and

485 the outside environment. Thus, genes involved in compatible solute synthesis would be expected

486 to be up-regulated in cells experiencing osmotic or matric stresses [26]. Previous studies have

487 suggested that the leaf surface is often sufficiently dry that compatible solute synthesis is needed

488 to combat matric stress $[44,45]$. However, since this experiment was performed on filters placed

489 on nutrient agar, the water status of cells upon such a surface is uncertain. While one might

490 imagine such a surface to be a drier habitat than what cells suspended in the corresponding broth

491 medium might experience, it is likely that this surface would be moister than leaf surfaces

492 typically are. The disruption of the gel matrix that would occur by application of a filter on an

493 agar surface might release free water in a process known as syneresis [46]. Indeed, filters

494 immediately appeared transiently wet after application to the agar surface. While the leaf surface

495 is composed of a waxy, hydrophobic cuticle to prevent the release of water vapor [47, 48], a

496 membrane filter is by definition quite porous and would enable the movement of water onto the

497 filter surface where it could wet cells.

498 The down-regulation of genes encoding carbohydrate metabolism and transport, organic acid

499 metabolism and transport, and amino acid metabolism and transport (Table 2) upon

500 immobilization of cells was surprising. While we expected nutrients to move from the agar

501 matrix through the filter to the attached cells on top, it is likely that the rate at which nutrients are

502 replenished by this diffusional process would be slower than that occurring during mixing of

503 cells in a planktonic state in the corresponding shaken broth medium. It might thus be expected

504 that the immobilized cells could experience a locally more nutrient-limited environment than 
505 those of the planktonic cells. The common observation of slower growth of bacteria on the

506 surface of membranes placed on agar media than in shaken broth of the same composition also

507 supports such a model. Interestingly it has been noted that many genes in $P$. syringae involved in

508 amino acid metabolism and transport were repressed on the leaf surface [26].

509 Surprisingly, genes involved in phytotoxin synthesis and transport as well as many other genes

510 enabling production of other secondary metabolites were repressed on the filter surface (Table

511 2). Such compounds often act as virulence factors. We had expected that contact with a surface

512 would serve as a cue to $P$. syringae that it had encountered a potential host plant after

513 transitioning from a planktonic existence, and thus one would have expected such compounds to

514 be expressed. However, the large majority of $P$. syringae cells in the phyllosphere of healthy

515 leaves usually occur only as epiphytes on the surface of leaves rather than within the apoplast

516 [49]. Thus, when P. syringae colonizes the leaf surface it seldom interacts directly with living

517 plant cells and thus would not be expected to benefit from the production of various toxins.

518 Rather, such toxins would prove beneficial only after it had entered the leaf apoplast [34] and

519 their expression is highly elevated in the apoplast, but not on the leaf surface [26]. We

520 hypothesize that a secondary signal, indicative of conditions within the leaf, and not a surface

521 itself, is required for expression of such virulence factors. Arbutin and other compounds that are

522 not common on leaf surfaces have also been shown to be required for the induction of toxins

523 such as syringomycin in P. syringae [50]. Such secondary cues would prevent the costly

524 production of toxins when they would not be beneficial.

525 One of the few studies that have examined gene expression in bacterial cells soon after surface

526 attachment assessed gene expression in Escherichia coli strain CSH50 by microarray analysis

527 one, four, and eight hours after planktonic cells had attached to mannose agarose beads 
528 submerged in cultures [13]. This study revealed that changes in gene expression upon

529 immobilization of cells were both very rapid and often transient as many genes that had become

530 induced by one hour of surface attachment exhibited subsequent decreases in expression by four

531 hours of attachment. By eight hours of attachment many of these genes exhibited continued

532 decreases in expression while others only then became induced. Interestingly, we found similar

533 functional genes in $P$. syringae to be up-regulated soon after cells attached to a surface, including

$534 e m r B$ encoding a drug resistance transporter as well as other genes in this operon, and $g \ln H$,

535 involved in the transport of glutamine (Fig S4). The gene marA, involved in resistance to a

536 variety of antimicrobial compounds and $\operatorname{mar} R$ encoding its regulator as well as $\operatorname{ahpF}$, grxA, and

$537 k a t G$ and their regulator $o x y R$, collectively mediating oxidative stress tolerance were also up-

538 regulated in both systems (Fig S4). Genes involved in attachment and DNA repair were up-

539 regulated in response to attachment in both systems as well. Taken together, these results suggest

540 that both $E$. coli and $P$. syringae experience chemical stresses to which these defensive responses

541 are necessary soon after mobilization on surfaces. Alternatively, surface attachment is taken as a

542 cue to alert cells that they have arrived at a surface upon which such chemical stresses could be

543 anticipated. E. coli can experience chemical stresses associated with innate resistance responses

544 of animal cells [51] as well as plants [37, 39]. Likewise, P. syringae experiences defensive

545 chemicals as well as oxidative stress in both compatible and incompatible host plants [38, 52]

546 and induces defensive genes in response [26]. Our work suggests that many such traits might be

547 induced in P. syringae merely upon contact with the leaf surface itself. Thus, stress response

548 traits, presumably less important in an open, aquatic environment, are rapidly induced in both $P$.

549 syringae and E. coli. when host defenses might otherwise prove lethal. 
550 In another study, Siryaporn et al. [31] examined rapid differential expression of genes in

551 Pseudomonas aeruginosa UCBPP-PA14 one hour after attaching to a surface, finding that pilY1

552 plays a central role in its surface-dependent induction of virulence and that the expression of

553 many genes was rapidly changed. Despite finding little surface-mediated induction of this

554 homolog in P. syringae, the homologs of several other genes were found to be induced in $P$.

555 syringae (Figs S4, S5, S6, S7). Among these genes were those involved in flagellar synthesis and

556 motility. A variety of genes involved in cell wall synthesis also exhibited similar regulatory

557 responses to surfaces in both bacteria. The genes $\operatorname{mra} Y$ and $m u r A$, involved in peptidoglycan

558 synthesis, as well as $\operatorname{lp} x C$ and $\operatorname{lp} x D$, involved in LPS synthesis, were induced upon

559 immobilization of both $P$. syringae and $P$. aeruginosa (Fig S7), suggesting that cell wall damage

560 is common upon interacting with surfaces $[8,30,53]$. Likewise, ispZ, involved in cell division,

561 was also induced on surfaces in both studies (Fig S7). It thus appears that there are many

562 common features of the initial interaction of a variety of bacteria taxa with surfaces, and that the

563 nature of the surface with which they interact may play a subservient role in dictating such

564 responses. A better understanding of the initial interaction of bacteria with surfaces hopefully

565 will lead to better manage their interactions in both agricultural and biomedical settings [12].

\section{Acknowledgements}

568 We thank Kevin Hockett and Gwyn Beattie for providing the gene functional category

569 assignments for Pseudomonas syringae B728a. We also thank Rebecca Mackelprang and Ke Bi

570 for their assistance with R to analyze RNA sequencing results. We also thank Steven Ruzin and

571 Denise Schichnes for assistance in Biological Imaging. This work used the Vincent J. Coates 
572 Genomics Sequencing Laboratory at UC Berkeley, supported by NIH S10 OD018174

573 Instrumentation Grant.

\section{References}

1. Morris CE, Sands DC, Vinatzer BA, Glaux C, Guilbaud C, Buffière A, et al. The life history of the plant pathogen Pseudomonas syringae is linked to the water cycle. ISME J. 2008; 2(3): 321-334.

2. Leveau JH, Lindow SE. Appetite of an epiphyte: quantitative monitoring of bacterial sugar consumption in the phyllosphere. Proc Natl Acad Sci U S A. 2001; 98(6): 34463453.

3. Lindow SE, Brandl MT. Microbiology of the phyllosphere. Appl Environ Microbiol. 2003; 69(4): 1875-1883.

4. Berti AD, Greve NJ, Christensen QH, Thomas MG. Identification of a biosynthetic gene cluster and the six associated lipopeptides involved in swarming motility of Pseudomonas syringae pv. tomato DC3000. J Bacteriol. 2007; 189(17): 6312-6323.

5. Burch AY, Zeisler V, Yokota K, Schreiber L, Lindow SE. The hygroscopic biosurfactant syringafactin produced by Pseudomonas syringae enhances fitness on leaf surfaces during fluctuating humidity. Environ Microbiol. 2014; 16(7): 2086-2098.

6. Hernandez MN, Lindow SE. Pseudomonas syringae increases water availability in leaf microenvironments via production of hygroscopic syringafactin. Appl Environ Microbiol. 2019; 85: e01014-19.

7. Burch AY, Browne PJ, Dunlap CA, Price NP, Lindow SE. Comparison of biosurfactant detection methods reveals hydrophobic surfactants and contact-regulated production. Environ Microbiol. 2011; 13(10): 2681-2691.

8. Chang CY. Surface sensing for biofilm formation in Pseudomonas aeruginosa. Front Microbiol. 2008; 8(2671). doi: 10.3389/fmicb.2017.02671.

9. Kearns DB. A field guide to bacterial swarming motility. Nat Rev Microbiol. 2010; 8: 634-644.

10. O’Toole GA, Wong GCL. Sensational biofilms: surface sensing in bacteria. Curr Opin Microbiol. 2016; 30: 139-146. 
11. Petrova O, Sauer K. Sticky situations: key components that control bacterial surface attachment. J Bacteriol. 2012; 194: 2413-2425.

12. Tuson HH, Weibel DB. Bacteria-surface interactions. Soft Matter. 2013; 9(18): 43684380 .

13. Bhomkar P, Materi W, Semenchenko V, Wishart DS. Transcriptional response of E. coli upon FimH-mediated fimbrial adhesion. Gene Regul Syst Bio. 2010; 4: 1-17.

14. King E, Ward M, Raney D. Two simple media for the demonstration of pyocyanin and fluorescin. J Lab Clin Med. 1954; 44(2): 301-307.

15. Afgan E, Baker D, Batut B, van den Beek M, Bouvier D, Čech M, et al. The Galaxy platform for accessible, reproducible and collaborative biomedical analyses: 2018 update. Nucleic Acids Res. 2018; 46: W537-W544.

16. Bolger AM, Lohse M, Usadel B. Trimmomatic: A flexible trimmer for Illumina sequence data. Bioinform. 2014; 30(15): 2114-20.

17. Feil H, Feil WS, Chain P, Larimer F, DiBartolo G, Copeland A, et al. Comparison of the complete genome sequences of Pseudomonas syringae pv. syringae B728a and pv. tomato DC3000. Proc Natl Acad Sci U S A. 2005; 102(31): 11064-11069.

18. Patro R, Duggal G, Love MI, Irizarry RA, Kingsford C. Salmon provides fast and biasaware quantification of transcript expression. Nat Methods. 2017; 14: 417-419.

19. Robinson MD, McCarthy DJ, Smyth GK. edge R: a Bioconductor package for differential expression analysis of digital gene expression data. Bioinform. 2010; 26(1): 139-140.

20. Robinson MD, Oshlack A. A scaling normalization method for differential expression analysis of RNA-seq data. Genome Biol. 2010; 11: R25. doi: 10.1186/gb-2010-11-3-r25.

21. R Core Team. R: A language and environment for statistical computing. R Foundation for Statistical Computing, Vienna, Austria. 2020; https:/www.R-project.org/.

22. Castillo-Davis CI, Hartl DL. GeneMerge - post-genomic analysis, data mining, and hypothesis testing. Bioinform. 2003; 19(7): 891-892.

23. Bonferroni CE. Teoria statistica delle classi e calcolo delle probabilit `a. Pubblicazioni del R Istituto Superiore di Scienze Economiche e Commerciali di Firenze. 1936; 8:3-62.

24. Benjamini Y, Hochberg Y. Controlling the false discovery rate: a practical and powerful approach to multiple hypothesis testing. J R Stat Soc B. 1995; 57:289-300. 
25. Loper JE, Lindow SE. Lack of evidence for in situ fluorescent pigment production by Pseudomonas syringae pv. syringae on bean leaf surfaces. Phytopathol. 1987; 77: 14491454.

26. Yu X, Lund SP, Scott RA, Greenwald JW, Records AH, Nettleton D, et al. Transcriptional responses of Pseudomonas syringae to growth in epiphytic versus apoplastic leaf sites. Proc Natl Acad Sci U S A. 2013; E425-E434.

27. Dötsch A, Eckweiler D, Schniederjans M, Zimmermann A, Jensen V, Scharfe M, et al. using RNA sequencing. PLOS ONE. 2012; 7(2): e31092. doi: 10.1371/journal.pone.0031092.

28. Hickman JW, Tifrea DF, Harwood CS. A chemosensory system that regulates biofilm formation through modulation of cyclic diguanylate levels. Proc Natl Acad Sci U S A. 2005; 102(40): 14422-14427.

29. Pringent-Combaret C, Vidal O, Dorel C, Lejeune P. Abiotic surface sensing and biofilmdependent regulation of gene expression in Escherichia coli. J Bacteriol. 1999; 181(19): 5993-6002.

30. Otto K, Silhavy TJ. Surface sensing and adhesion of Escherichia coli controlled by the Cpx-signaling pathway. Proc Natl Acad Sci U S A. 2002; 99(4): 2287-2292. Pseudomonas aeruginosa virulence. Proc Natl Acad Sci U S A. 2014; 111(47): 1686016865.

32. Wood LF, Leech AJ, Ohman DE. Cell wall-inhibitory antibiotics activate the alginate biosynthesis operon in Pseudomonas aeruginosa: Roles of $\sigma^{22}$ (AlgT) and the AlgW and Prc proteases. Mol Microbiol. 2006; 62(2): 412-426.

33. Wood LF, Ohman DE. Use of cell wall stress to characterize $\sigma^{22}(\mathrm{AlgT} / \mathrm{U})$ activation by regulated proteolysis and its regulon in Pseudomonas aeruginosa. Mol Microbiol. 2009; 72(1): 183-201. bacterial diseases. Annu Rev Phytopathol. 2008; 46: 101-122. reproductive success at single-cell resolution. The ISME Journal. 2010; 4: 215-222.

36. Remus-Emsermann MNP, Tecon R, Kowalchuk GA, Leveau JHJ. Variation of local carrying capacity and the individual fate of bacterial colonizers in the phyllosphere. ISME J. 2012; 6: 756-765. 
37. Dublan MA, Ortiz-Marquez JCF, Lett L, Curatti L. Plant-adapted Escherichia coli show increased lettuce colonizing ability, resistance to oxidative stress and chemotactic response. PLOS ONE. 2014; 9(10): e110416. doi: 10.1371/journal.pone.0110416.

38. Fones H, Preston GM. Reactive oxygen and oxidative stress tolerance in plant pathogenic Pseudomonas. FEMS Microbiol Lett. 2012; 327(1): 1-8.

39. Kyle JL, Parker CT, Goudeau D, Brandl MT. Transcriptome analysis of Escherichia coli O157:H7 exposed to lysates of lettuce leaves. Appl Environ Microbiol. 2010; 76(5): 1375-1387.

40. Joyner DC, Lindow SE. Heterogeneity of iron bioavailability on plants assessed with a whole-cell GFP-based bacterial biosensor. Microbiol. 2000; 146: 2435-2445.

41. Temple TN, Stockwell VO, Loper JE, Johnson VB. Bioavailability of Iron to Pseudomonas fluorescens Strain A506 on Flowers of Pear and Apple. Phytopathol. 2004; 94: 1286-1294.

42. Dulla GM, Marco M, Quiñones B, Lindow SE. A closer look at Pseudomonas syringae as a leaf colonist. ASM News. 2005; 71: 469-475.

43. Haefele DM, Lindow SE. Flagellar motility confers epiphytic fitness advantages upon Pseudomonas syringae. Appl Environ Microbiol. 1987; 53: 2528-2533.

44. Axtell CA, Beattie GA. Construction and characterization of a $p r o U-g f p$ transcriptional fusion that measures water availability in a microbial habitat. Appl Environ Microbiol. 2002; 68(9): 4604-4612.

45. Beattie GA. Water relations in the interaction of foliar bacterial pathogens with plants. Annu Rev Phytopathol. 2011; 49: 533-55.

46. Banerjee S, Bhattacharya S. Compressive textural attributes, opacity and syneresis of gels prepared from gellan, agar and their mixtures. J Food Eng. 2011; 102: 287-292.

47. Hess DF, Foy CL. Interaction of surfactants with plant cuticles. Weed Technol. 2000; 14(4): 807-813. syringae - a pathogen, ice nucleus, and epiphyte. Microbiol Mol Bio Rev. 2000; 64(3): 624-653. relation to pathogenicity and potential for growth within the leaf. Appl Environ Microbiol 1999; 65: 1435-1443. 

filters. surface (black).

50. Mo YY, Gross DC. Plant signal molecules activate the $s y r B$ gene, which is required for syringomycin production by Pseudomonas syringae pv. syringae. J Bacteriol. 1991; 173(18): 5784-5792.

51. Tagkopoulos I, Liu YC, Tavazoie S. Predictive behavior within microbial genetic networks. Science. 2008; 320: 1313-1317.

52. Alcalde-Rico M, Hernando-Amado S, Blanco P, Martinez JL. Multidrug efflux pumps at the crossroad between antibiotic resistance and bacterial virulence. Front Microbiol. 2016; 7: 1483. doi: 10.3389/fmicb.2016.01483.

53. Vogt SL, Raivio TL. Just scratching the surface: an expanding view of the Cpx envelope stress response. FEMS Microbiol Lett. 2012; 326(1): 2-11.

\section{Supporting Information}

Figure S1. Number of sequencing reads. Number of sequencing reads obtained for the various replicate samples of Pseudomonas syringae B728a cells recovered 2 hours after application to filters or from cells in broth cultures used to inoculate the filters.

Figure S2. Distribution of number of sequencing reads. Distribution of number of sequencing reads assigned to a given gene following RNA sequencing of Pseudomonas syringae B728a cells recovered 2 hours after application to filters or from cells in broth cultures used to inoculate the

\section{Figure S3. Smear plot documenting the differential expression of genes in Pseudomonas} syringae B728a. Smear plot documenting the differential expression of genes in Pseudomonas syringae B728a 2 hours after inoculation onto filter surfaces compared to that in planktonic cells as a function of their levels of expression. Shown is the proportion of the genes that were differentially expressed on the filter surface (red) and those not differentially expressed on a 
732 Figure S4. Comparison of differential expression of homologs of genes in Pseudomonas

733 syringae B728a and in $\boldsymbol{E}$. coli CSH50. Comparison of differential expression of homologs of

734 genes in Pseudomonas syringae B728a 2 hours after application to membrane surfaces and of $E$.

735 coli CSH50 1 hour after attachment to mannose agarose beads in the study of Bhomkar et al.

736 (2010). Differential up-regulation of genes is shown in green, down-regulation is depicted in red,

737 and no change in expression is shown in yellow.

738 Figure S5. Relationship between magnitude (fold-change) of differential expression of gene

739 homologs in Pseudomonas syringae B728a and in E. coli CSH50. Relationship between

740 magnitude (fold-change) of differential expression of gene homologs in Pseudomonas syringae

741 B728a 2 hours after transfer to a filter surface and that of E. coli CSH50 1 hour after attachment

742 on agarose beads in the study of Bhomkar et al. (2010).

743 Figure S6. Comparison of the number of gene homologs in Pseudomonas syringae B728a

744 and in Pseudomonas aeruginosa UCBPP-PA14. Comparison of the number of gene homologs

745 in Pseudomonas syringae B728a (blue) and Pseudomonas aeruginosa UCBPP-PA14 (red) that

746 were up-regulated when transferred to filter surfaces for 2 hours or were attached to a glass

747 surface for 1 hour in the study of Siryaporn et al. (2014), respectively. Using the hypergeometric

748 distribution, this overlap was determined to be significant with a p-value of 1.65E-05 (Castillo-

749 Davis and Hartl, 2003).

750 Figure S7. Comparison of differential expression of homologs of genes in Pseudomonas

751 syringae B728a and in Pseudomonas aeruginosa UCBPP-PA14. Comparison of differential

752 expression of homologs of genes in Pseudomonas syringae B728a 2 hours after application to

753 membrane surfaces and of Pseudomonas aeruginosa UCBPP-PA14 wild-type cells attached to a

754 glass surface for 1 hour in the study of Siryaporn et al. (2014). Differential up-regulation of 
bioRxiv preprint doi: https://doi.org/10.1101/2020.10.20.346825; this version posted October 21, 2020. The copyright holder for this preprint (which was not certified by peer review) is the author/funder, who has granted bioRxiv a license to display the preprint in perpetuity. It is made available under aCC-BY 4.0 International license.

755 genes is shown in green, down-regulation is depicted in red, and no change in expression is

756 shown in yellow. 


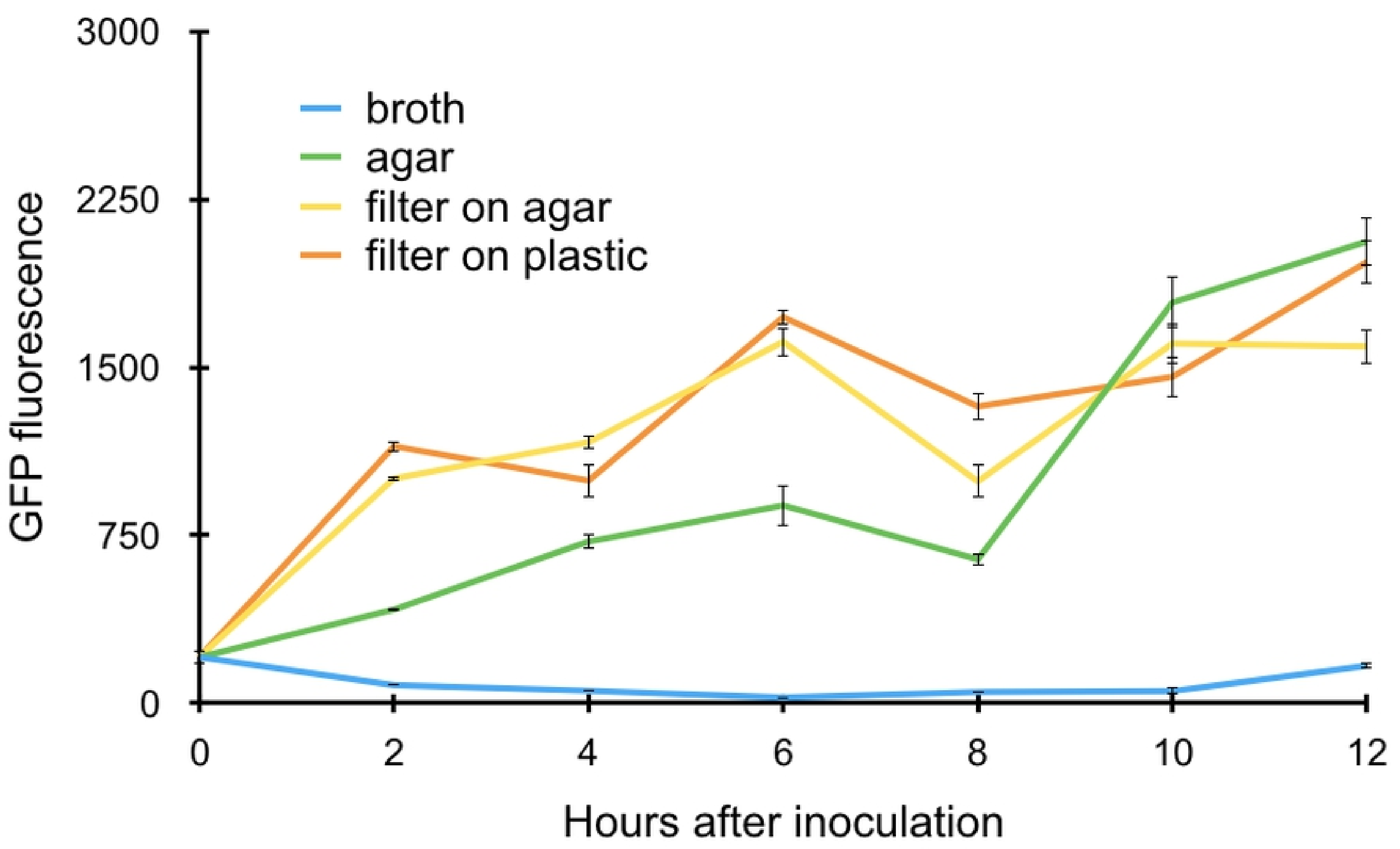

Figure 


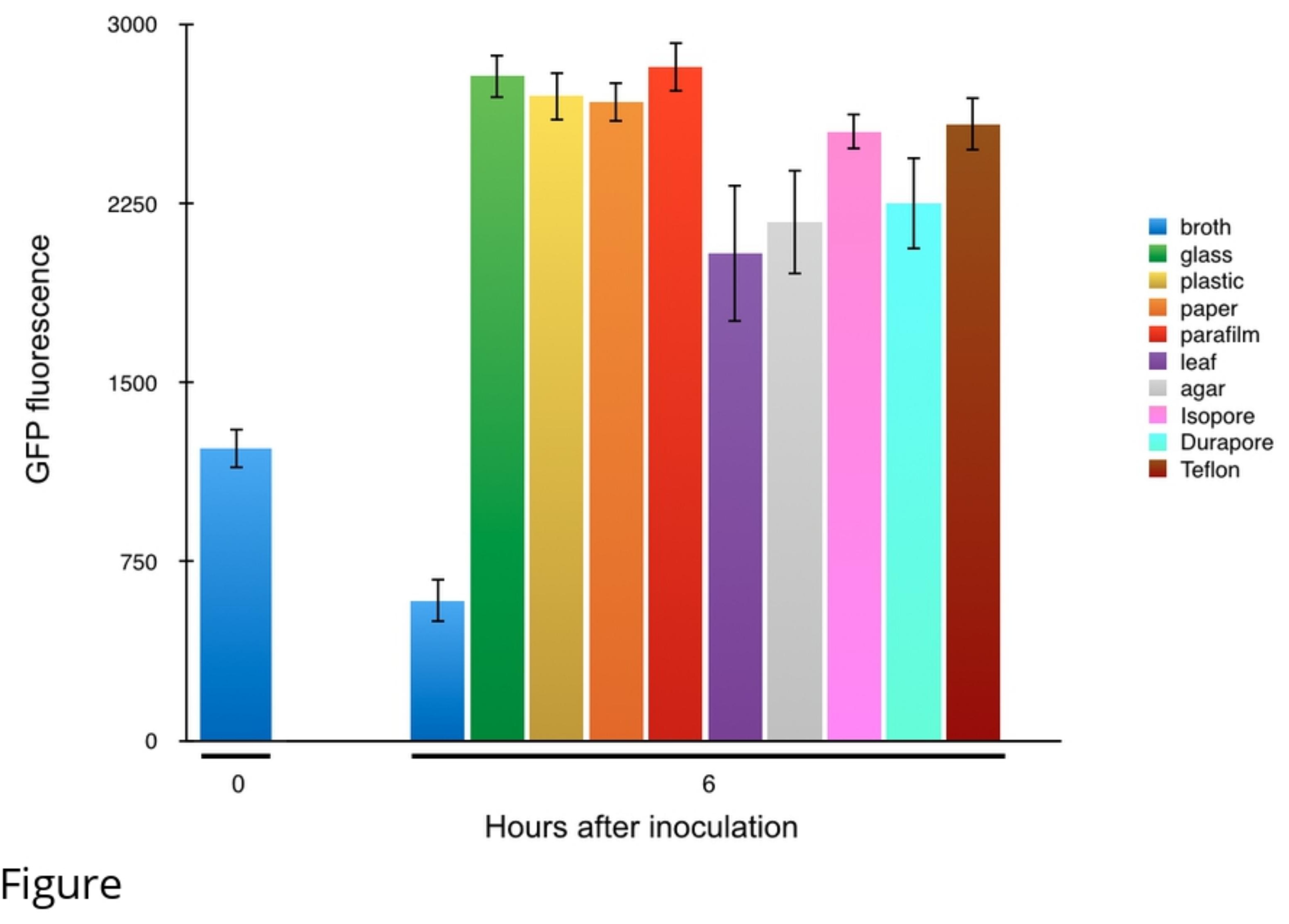




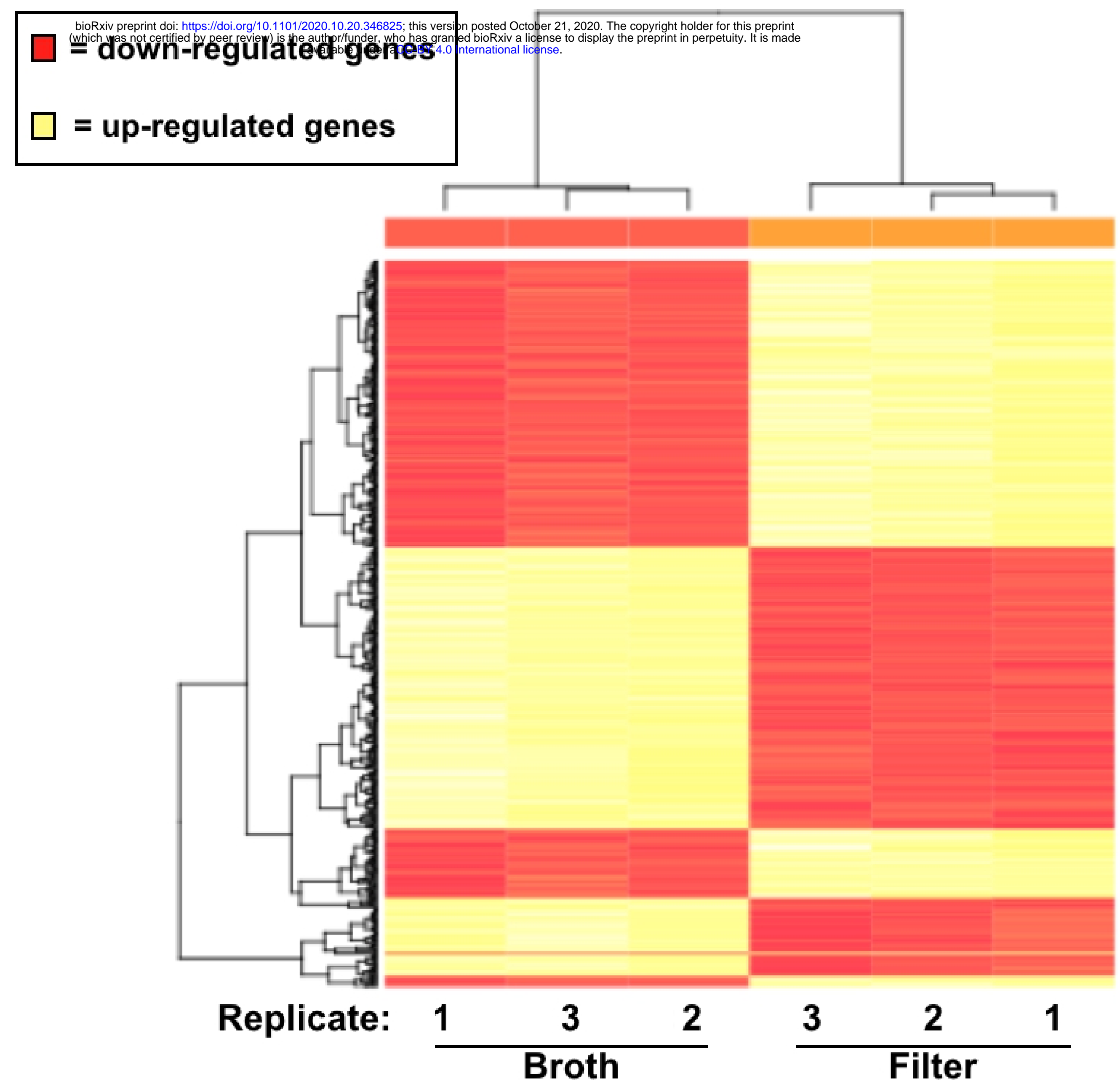

Figure 


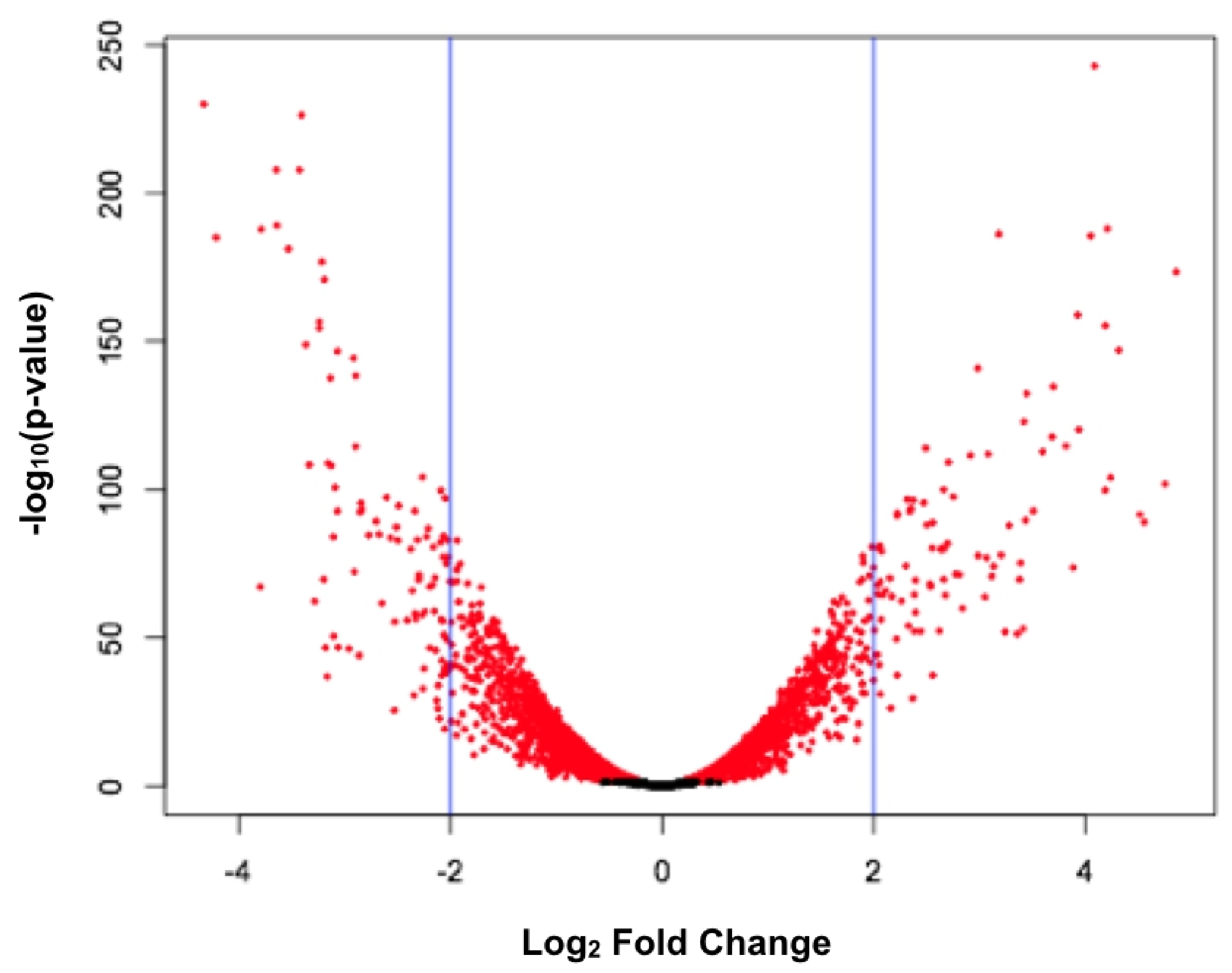

Figure 